\title{
The saccadic re-centering bias is associated with activity changes in the human superior colliculus
}

\author{
Ruth M. Krebs' ${ }^{1,2}$, Mircea A. Schoenfeld ${ }^{2,3,4}$, Carsten N. Boehler ${ }^{1,4}$, Allen W. Song ${ }^{5}$ and Marty G. Woldorff1,6 \\ ' Center for Cognitive Neuroscience, Duke University, Durham, NC, USA \\ 2 Department of Neurology, Otto-von-Guericke-University, Magdeburg, Germany \\ 3 Kliniken Schmieder, Allensbach, Germany \\ ${ }^{4}$ Department of Behavioral Neurology, Leibniz-Institute for Neurobiology, Magdeburg, Germany \\ ${ }_{5}^{5}$ Brain Imaging and Analysis Center, Duke University, Durham, NC, USA \\ ${ }^{6}$ Department of Psychiatry, Duke University, Durham, NC, USA
}

Edited by:

Chris Rorden, Georgia Institute of Technology, USA

\section{Reviewed by:}

Árni Kristjánsson, University of Iceland, Iceland

Paul Corballis, Georgia Institute of Technology, USA

${ }^{*}$ Correspondence:

Ruth M. Krebs, Center for Cognitive Neuroscience, B203 Levine Science Research Center, Duke University, Durham, NC 27708, USA.

e-mail: ruth.krebs@duke.edu
Being able to effectively explore our visual world is of fundamental importance, and it has been suggested that the straight-ahead gaze (primary position) might play a special role in this context. We employed fMRI in humans to investigate how neural activity might be modulated for saccades relative to this putative default position. Using an endogenous cueing paradigm, saccade direction and orbital starting position were systematically manipulated, resulting in saccades toward primary position (centripetal) and away from primary position (centrifugal) that were matched in amplitude, directional predictability, as well as orbital starting position. In accord with earlier research, we found that $\mathrm{fMRI}$ activity in the superior colliculus (SC), as well as in the frontal eye fields and the intraparietal sulcus, was enhanced contralateral to saccade direction across all saccade conditions. Furthermore, the $\mathrm{SC}$ exhibited a relative activity decrease during re-centering relative to centrifugal saccades, a pattern that was paralleled by faster saccadic reaction times. In contrast, activity within the cortical eye fields was not significantly modulated during re-centering saccades as compared to other saccade types, suggesting that the re-centering bias is predominantly implemented at a subcortical rather than cortical processing stage. Such a modulation might reflect a special coding bias facilitating the return of gaze to a default position in the gaze space in which retinotopic and egocentric reference frames are aligned and from which the visual world can be effectively explored.

Keywords: superior colliculus, fMRI, eye movement, re-centering bias, cortical eye fields

\section{INTRODUCTION}

The execution of eye movements to objects or locations of interest is carried out by a complex network of cortical and subcortical brain regions enabling a precise alignment of the observer's fovea with the object of interest (for a review see Wurtz and Albano, 1980; Sparks, 2002). One of the crucial structures both for the reflexive orienting toward salient stimuli and for the initiation of voluntary saccadic eye movements is the superior colliculus (SC), a small layered structure that forms part of the tectum of the midbrain. The SC receives multiple cortical and subcortical inputs and represents the final relay structure for eye-movement execution (Fries, 1984; Sparks et al., 2000; Schiller and Tehovnik, 2001; Sparks, 2002; Sommer and Wurtz, 2008). Moreover, the SC has been shown to be involved in the selection of future gaze locations based on a visual salience map (e.g., Fecteau and Munoz, 2006; Boehnke and Munoz, 2008), as well as in covert shifts of attention in the absence of eye movements (e.g., Ignashchenkova et al., 2004).

Among its wide range of connections, the SC receives prominent input from two cortical regions that are linked to both overt saccades and covert attentional shifts: the frontal eye fields (FEF) located in the premotor cortex (Paus, 1996; Blanke et al., 2000; Koyama et al., 2004) and an area within the intraparietal sulcus (IPS; encompassing BA7 and BA40) considered to be the human equivalent of the primate lateral intraparietal area (LIP, Muri et al.,
1996; Luna et al., 1998; Koyama et al., 2004). Numerous studies in animals and humans have indicated that the IPS is part of the direct visual pathway for the generation of reflexive saccades and seems to be involved in the transformation of saccade vector coordinates that will finally be transmitted to the SC (e.g., Duhamel et al., 1992; Gaymard et al., 1998). In turn, FEF has been specifically associated with target selection during the generation of voluntary saccades (e.g., Sommer and Tehovnik, 1997; Mort et al., 2003). Beyond the generation of saccadic eye movements, both the FEF and the IPS have also been associated with the covert orienting of attention in a wide range of paradigms, consistent with the view that these two functions are supported by overlapping neural systems (Corbetta and Shulman, 2002).

One important property that appears to be shared by saccadeprocessing regions, including the SC, the FEF, and the IPS, is the contralateral representation of saccade targets based on the underlying retinotopic (eye-centered) reference frame, thus resulting in enhanced activity in the hemisphere contralateral to the targeted visual field. This contralateral predominance (also referred to as contralateral bias) has been demonstrated in a large number of animal studies using single-unit recordings and micro-stimulation measures targeting the SC (Goldberg and Wurtz, 1972; Robinson, 1972; Schiller and Stryker, 1972; Kustov and Robinson, 1996; Moschovakis, 1996; Schiller and Tehovnik, 2001; Ignashchenkova 
et al., 2004; Field et al., 2008) and the FEF (Bruce and Goldberg, 1985; Schall, 1995; Tehovnik and Slocum, 2000; Crapse and Sommer, 2009). In the monkey LIP, although topographically organized, most neurons seem to exhibit a relatively small contralateral bias (Blatt et al., 1990; Platt and Glimcher, 1997). In humans, the contralateral representation of stimulus input and saccade targets has been mostly demonstrated at the cortical level, including FEF, occipital cortex, and IPS (Sereno et al., 2001; Astafiev et al., 2003; Schluppeck et al., 2005; Silver et al., 2005; Kastner et al., 2007; Serences and Yantis, 2007; Curtis and Connolly, 2008; Ikkai and Curtis, 2008; Konen and Kastner, 2008). With the increasing quality of neuroimaging techniques, however, studies have started to investigate the functional contralateral organization of the human SC during visual stimulation and covert attention shifts (DuBois and Cohen, 2000; Schneider and Kastner, 2005, 2009; Wall et al., 2009) and during saccadic eye movements (Krebs et al., 2010).

The retinotopic reference frame underlying this contralateral representation is separated into the left and right visual field by the vertical meridian that intersects visual fixation. Considering this relationship within an eye-centered reference frame, it might be inferred that the representation of saccade targets is completely independent of the eye position relative to the head, and thus, given a head-restrained setting, that saccade vectors and parameters can be fully described in retinotopic coordinates. However, there is evidence that saccade processing is not independent of the egocentric (head-centered) reference frame. A number of behavioral studies in humans (Laurutis and Robinson, 1986; Zambarbieri et al., 1995; Fuller, 1996), as well as studies in animals (Albano and Wurtz, 1982; McIlwain, 1986; Pare and Munoz, 1996), have shown that saccades toward the center of straight-ahead gaze (primary position) are facilitated as compared to saccades to other gaze locations, as measured by saccadic reaction times (SRT). It has been proposed that this "re-centering bias" (also referred to as centripetal bias) might subserve the efficient coordination of eye- and head-movements by providing a common default position (Fuller, 1996; Pare and Munoz, 2001; Tatler, 2007). This primary position, in which the retinotopic and egocentric reference frame are aligned, is considered to be a particularly efficient starting point for exploring the visual environment in an energy-efficient way (Vitu et al., 2004; Tatler, 2007; Kardamakis and Moschovakis, 2009; Durand et al., 2010).

The neural processes underlying the behavioral findings of a re-centering bias, however, are not well understood. In addition to the well described retinotopic saccade-target representation, such a modulation would require information about the current eye position relative to the current head-position being available to the relevant saccade-controlling brain regions. Indeed, based on studies investigating saccade-related SC activity in animals, there is evidence that the SC might be involved in the processing of eyeposition signals (McIlwain, 1986; Van Opstal et al., 1995; Pare and Munoz, 2001; Campos et al., 2006) and that such a signal might contribute to the facilitation of re-centering the eyes to the primary position. More specifically, it has been proposed that saccades of identical direction and amplitude require different levels of innervation at the motor plant depending on the orbital deviation from primary position and that this might be reflected in a dynamic adaptation of the neuronal activity in the SC (Van Opstal et al., 1995; Campos et al., 2006). With regard to the role of human SC in this context, we recently observed differential activity changes in the SC for saccades toward (centripetal) and away (centrifugal) from primary position (Krebs et al., 2010). However, in our previous paradigm, centripetal (i.e., re-centering) and centrifugal saccades differed in their directional predictability. In addition, since the fMRI acquisition in that study was limited to a small volume optimized for the brainstem, the data provided no information about cortical eye fields, i.e., FEF and IPS, and their possible modulation during re-centering saccades.

In the present study we employed fMRI during a paradigm in which subjects performed endogenously cued $7^{\circ}$-saccades to the left and right between five target positions along the horizontal meridian (see Figure 1). Saccade vectors were oriented toward (centripetal) or away from (centrifugal) the primary central position (orientation factor), starting from positions that were either proximal or distal to the primary gaze position (proximity factor). In addition to the contralateral coding of saccade direction (contralateral predominance factor), this design allowed us to probe the processing level at which the facilitation of saccades toward the primary position primarily occurs.

On the basis of earlier work in animals and humans, we hypothesized that the re-centering bias would be specifically reflected by activity changes within the SC, due to its role in saccade programming and its capacity to incorporate both eye- and head-position information (Van Opstal et al., 1995; Campos et al., 2006). Importantly, by using endogenous cues, the present paradigm enabled us to isolate neural responses in the absence of salient peripheral targets which strongly modulate SC activity (e.g., Boehnke and Munoz, 2008), and may have tended to dilute the neural processing activity related to the re-centering bias. Furthermore, we expected relatively little modulation related to the re-centering bias in cortical areas (FEF and IPS). More specifically, given that all saccades in the current paradigm were endogenously cued, including the re-centering saccades, they would be likely to engage similar levels of top-down cortical control during cue interpretation and voluntary target selection before the saccade-initiation command is passed on to the SC. At this later processing stage, the SC could then efficiently facilitate saccades toward the primary position by incorporating eye- and head-position information.

\section{MATERIALS AND METHODS FMRI SUBJECTS AND PARADIGM}

Sixteen healthy right-handed subjects participated in the fMRI study (mean age \pm standard deviation (SD): $25 \pm 4.6$, eight female). Four of initially 20 subjects had to be excluded based on their performance in the saccade task (two subjects) or due to technical difficulties (two subjects). Participants gave written informed consent before the experiment in accordance with the Duke Medical Center Institutional Review Board for human subjects.

We used an event-related fMRI saccade paradigm that manipulated saccade direction (left vs. right), the orientation of the saccade vector (centrifugal vs. centripetal), and the proximity of the saccade vector (proximal vs. distal) relative to the primary position. At the beginning of each experimental run, subjects saw an instruction screen for $6 \mathrm{~s}$ that displayed the assignment of the color cues (e.g., green $=$ saccade to the left, blue $=$ saccade to the right, red $=$ maintain fixation). The color-direction mapping was consistent throughout 
A

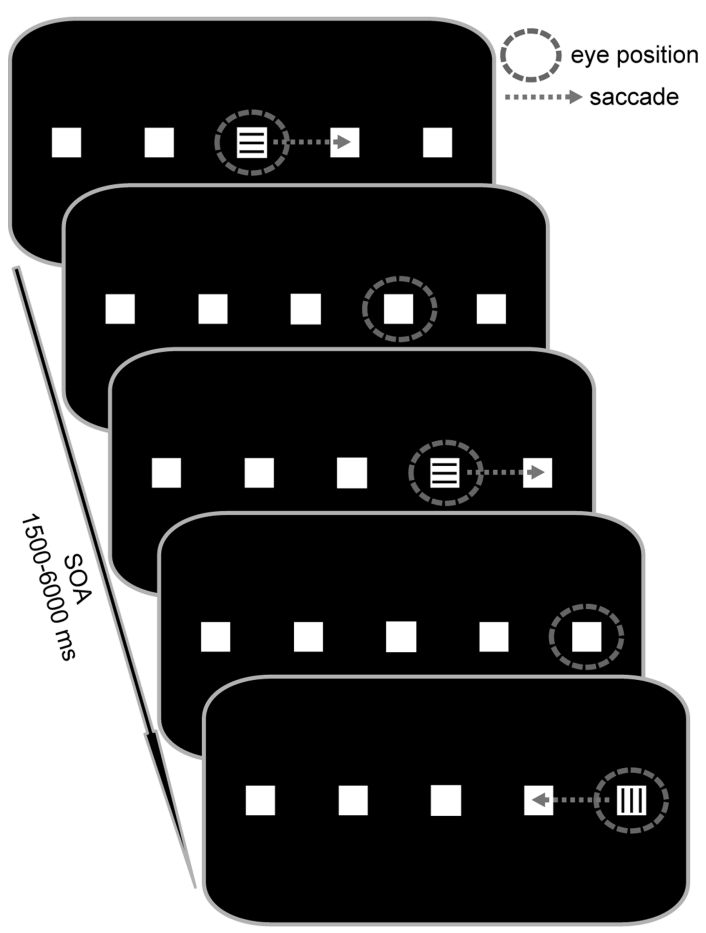

FIGURE $\mathbf{1}$ |Trial structure and study design. (A) Subjects performed saccades from square to square along the horizontal meridian. Instructional color cues (300 ms duration) at the currently foveated square signaled the direction of the next saccade (e.g., "blue = right" vs. "green = left"; here represented by horizontal vs. vertical lines) or indicated to maintain fixation (e.g., "red = stay"). After each saccade, the subjects' gaze remained at the new square until the next saccade was triggered by a color cue. The stimulus onset asynchrony (SOA) was jittered between 1.5 and $6 \mathrm{~s}$ for all saccade and fixation cues. (B) Saccade vectors were oriented either toward (centripetal) or away from (centrifugal) the

the experiment for each subject, but was counterbalanced across subjects. The instructions were followed by the default stimulus display consisting of five white squares (each $0.5^{\circ} \times 0.5^{\circ}$ of visual angle) on a black background, with the central square located at the center of the screen and with an even between-square spacing of $7^{\circ}$ (Figure 1A). In regards to this layout, we will refer to the central position as being at position 0 , the adjacent ones as being at position 1 on the left or right, and most eccentric ones as being at position 2 on the left or right.

At the beginning of each run, subjects fixated the center square until a colored cue (duration $300 \mathrm{~ms}$ ) appeared at that position, with its color signaling to either execute the first $7^{\circ}$-saccade to the adjacent square in the indicated direction or to maintain fixation. After the eye movement was performed, the subjects' gaze remained at the new square until the next cue appeared at the newly foveated position. In fixation trials (e.g., as indicated by, for example, a red cue), the subjects' gaze remained at the same square until the following cue signaled a new eye movement. Saccade direction from all positions was randomized throughout each run, except from the $\pm 14^{\circ}$-position, which necessarily required a return to the neighboring more medial position. Subjects were asked to execute each saccade as quickly and as accurately as possible and to
B

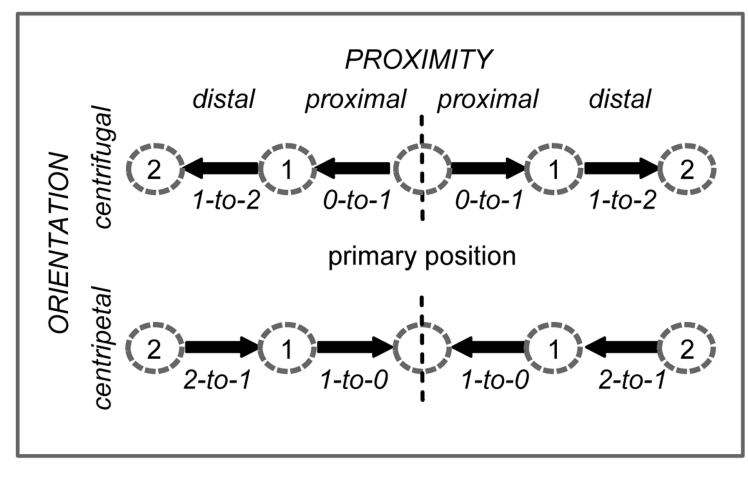

C

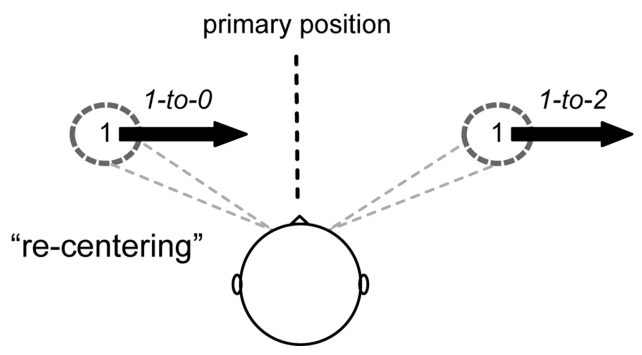

primary position (defined as head-centered straight gaze) and also differed in their proximity to the primary position (proximal vs. distal). The design thus resulted in four saccade trial types represented by vectors with specific start and end positions (start-to-end), i.e., proximal centrifugal (0-to-1), distal centrifugal (1-to-2), proximal centripetal (1-to-0), and distal centripetal (2-to-1), each of which could be performed to the left or right side. (C) Depending on the initial deviation of the eyes relative to primary position, rightward saccades of identical amplitude either re-center the eyes or move them further away from primary position.

minimize blinking. The timing of cue onsets was pseudo-randomly varied with a stimulus onset asynchrony (SOA) of 1.5-6 s (average $\mathrm{SOA}=2.5 \mathrm{~s}$ ) to allow for effective event-related blood-oxygen level-dependent (BOLD) response estimation (Hinrichs et al., 2000). The event-related design resulted in eight saccade conditions (Figure 1B), along with the cued fixation condition that served as a baseline in which an instructional cue was presented but no eye movement was performed.

Importantly, the employed design allowed us to compare centripetal saccades and centrifugal saccades (see Figure 1B: proximal centripetal 1 -to-0 vs. distal centrifugal 1-to-2) with identical shift amplitude, identical directional probability (i.e., the direction of the saccade was not predictable), and, importantly, with an equivalent pre-saccadic deviation from the primary position (as illustrated in Figure 1C). These saccades differed particularly in the orientation of their movement with respect to the primary position (centripetal vs. centrifugal) and were thus particularly well suited for investigating the neural correlates associated with the re-centering bias. The additional saccade conditions (see Figure 1B: proximal centrifugal 0-to-1 and distal centripetal 2-to-1) were mainly included to provide equal directional probabilities throughout the task. 


\section{fMRI DATA ACQUISITION}

Prior to actual scanning, subjects performed a 10-min training session to get familiarized with the task. Inside the scanner, subjects performed seven experimental runs, each of 7-min duration, resulting in a total of 112 trials in each saccade condition: left and right proximal centrifugal (0-to-1), left and right proximal centripetal back to primary position (1-to-0), left and right distal centrifugal (1-to-2), left and right distal centripetal (2-to-1), and a total of 70 fixation trials (i.e., 14 trials at each of the five squares). fMRI images were acquired using a 3 Tesla GE EXCITE HD scanner with an eightchannel head-coil array. Each functional run consisted of 290 images that were acquired in an axial slice orientation (30 slices, thickness $3 \mathrm{~mm}$ ) using an interleaved scanning order (inward spiral sequence with SENSE acceleration factor of $2, \mathrm{TR}=1500 \mathrm{~ms}$, TE $=25 \mathrm{~ms}$, FoV $=192 \mathrm{~mm}$, matrix size of $64 \times 64$ yielding an in-plane resolution of $3 \mathrm{~mm} \times 3 \mathrm{~mm}$ ). The first five time points of each run were discarded to allow a steady magnetization to be reached.

For each subject, a T1-weighted high-resolution whole-brain anatomical scan (3D FSPGR sequence, FoV $=256 \mathrm{~mm}$, yielding a voxel size of $1 \mathrm{~mm} \times 1 \mathrm{~mm} \times 1 \mathrm{~mm}$ ) was acquired to enable coregistration and normalization to a template brain. During the entire experiment, subjects were instructed to keep their head-position straight and remain still, which was monitored online and facilitated by passive padding inside the head coil. In order to control for the correct execution of the saccade task, eye movements were monitored online throughout all runs using an MR-compatible eyetracking system (Viewpoint, Arrington Research, Scottsdale, AZ, USA). Eye position was monitored at $30 \mathrm{~Hz}$ via an infrared camera that was mounted above the subject's right eye and calibrated at the beginning of the scanning session.

\section{fMRI DATA ANALYSIS}

Images were preprocessed and analyzed using the Statistical Parametric Mapping software package (SPM5; Wellcome Department of Imaging Neuroscience, University College, London, UK). Anatomical images were co-registered to the SPM template and spatially normalized. Functional images were corrected for acquisition delay, spatially realigned, and co-registered to the original T1-weighted image. After spatial normalization to a final voxel size of $2 \mathrm{~mm} \times 2 \mathrm{~mm} \times 2 \mathrm{~mm}$, functional images were smoothed with an isotropic 4-mm full-width half-maximum Gaussian kernel. Before model estimation, a high-pass temporal filter of $128 \mathrm{~s}$ was applied (Ashburner and Friston, 1999).

A two-stage model was used for statistical analysis (Friston et al., 1995). In the first stage, BOLD responses were modeled by delta functions at the stimulus onsets for the nine event types (left and right centrifugal 0 -to-1 saccades, left and right centripetal 1-to-0 saccades, left and right centrifugal 1-to-2 saccades, left and right centripetal 2-to-1 saccades, and fixation trials), which were then all convolved with a canonical hemodynamic response function (HRF). In order to optimize the signal estimation within the SC, as suggested by Wall et al. (2009) and Krebs et al. (2010), we used a version of the canonical HRF that peaked at $4.5 \mathrm{~s}$ instead of at the standard $6 \mathrm{~s}$. The resulting estimates together with the corresponding temporal and dispersion derivatives as well as six realignment parameters for each run formed covariates of a general linear model (GLM, Friston et al., 1995). Individual subjects' contrast images were entered into a random effects analysis using one-sample $T$-tests for voxel-wise comparisons (significance threshold $p<0.001$, voxel-extent threshold $k>15$ ). At the voxel-wise level a contrast for "all saccades vs. fixation" was calculated, which was used to establish the general saccade-related activity.

To investigate whether saccade-related regions were sensitive to the direction, the orientation, and the proximity of the saccade vector relative to the primary position, we performed a region of interest (ROI) analysis using the MarsBar analysis toolbox (Ollinger et al., 2001; Brett et al., 2002). ROIs were defined bilaterally as cubes centered at the local activity maxima derived from the conditionindependent voxel-wise comparison "all saccades vs. fixation" within $\mathrm{FEF}(8 \mathrm{~mm} \times 8 \mathrm{~mm} \times 8 \mathrm{~mm})$ and IPS $(8 \mathrm{~mm} \times 8 \mathrm{~mm} \times 8 \mathrm{~mm})$, as well as in the SC $(4 \mathrm{~mm} \times 4 \mathrm{~mm} \times 4 \mathrm{~mm})$. From these ROIs the mean parameter estimates (beta values) for each condition of interest, i.e., left and right for proximal centrifugal (0-to-1), proximal centripetal (1-to-0), distal centrifugal (1-to-2), and distal centripetal (2-to-1), were extracted. The contrast "all saccades vs. fixation" was chosen to allow for a condition-independent selection of the areas within SC, FEF, and IPS that were associated with general saccade generation.

For all ROIs (FEF, IPS, and SC), the extracted parameter estimates were collapsed across hemispheres, yielding activity that was contralateral to saccade direction (i.e., activity within left hemisphere during saccades to the right and vice versa) and ipsilateral to saccade direction (i.e., activity within left hemisphere during saccades to the left and vice versa), respectively. Data were then analyzed via repeated-measures analyses of variance (rANOVA), with the factors contralateral predominance (contralateral vs. ipsilateral), orientation (centrifugal vs.centripetal), and center proximity (proximal vs. distal). In order to evaluate the actual region-specific shape of the BOLD response, we extracted the activity time course for each ROI across all saccade conditions based on a shape-assumption-free finite-impulse-response (FIR) model. This indicated that, in accord with earlier reports (Ikkai and Curtis, 2008), the non-standard HRF with a somewhat earlier peak was in fact also effective for characterizing activity within FEF and IPS.

Based on our previous findings regarding differential saccaderelated activity in a frontal region associated with the default-mode network of attention (Krebs et al., 2010), we performed an additional ROI analysis in bilateral medial prefrontal cortex (mPFC). The center coordinates for the cubical ROIs $(4 \mathrm{~mm} \times 4 \mathrm{~mm} \times 4 \mathrm{~mm}$; left: $x, y, z=-6,44,10$; right: $x, y, z=12,38,-12)$ were derived from the contrast "fixation vs. all saccades" based on the standard HRF, yielding an unbiased ROI for assessing differential activation in the default-mode network (Raichle et al., 2001). The extracted parameter estimates (beta values) were collapsed across left and right mPFC and analyzed via rANOVA with factors orientation (centrifugal vs. centripetal) and center proximity (proximal vs. distal).

\section{ELECTRO-OCULOGRAM STUDY}

Although the eye-tracking signal acquired during the fMRI session can be used to quantify general task performance (e.g., the number of correct and erroneous saccades), online measures during fMRI scanning are often of limited use. Particularly, various sources of artifacts (e.g., eye-tracking disturbances caused by the onset of the fMRI gradients) lead to relatively low number of analyzable trials. Moreover, the relatively low temporal resolution of 
the fMRI eye-tracker (sampling rate of $30 \mathrm{~Hz}$ ) is not sufficient to provide a sensitive measure for small differences in SRTs between the conditions of interest. To compensate for these limitations, we derived saccade onset times by analyzing the electro-oculogram (EOG) channels recorded during an equivalent electroencephalographic (EEG) study that was performed outside the scanner in a sparsely lit electrically shielded chamber. Here, 16 subjects (mean age \pm SD: $22 \pm 2.6$, six female) performed the exact same task used in the fMRI study, with the only differences being a higher number of trials per condition (160) and shorter SOAs (randomly jittered between 1 and $1.5 \mathrm{~s}$ ). The EOG channels were continuously recorded with a band-pass filter of $0.01-100 \mathrm{~Hz}$ at a sampling rate of $500 \mathrm{~Hz}$ (SynAmps amplifiers Neuroscan; http:// www.neuroscan.com).

After saccade-locked segmentation of the horizontal EOG channel, trials containing artifacts (e.g., blinks) were removed from the data while clean trials were separated in correctly executed and erroneous saccades using the Brain Vision Analyzer 1.05 software (Brainproducts Co, Munich, Germany). Saccade onset latencies of correctly executed saccades were averaged for each experimental condition (left and right centrifugal 0 -to-1, left and right centripetal 1-to-0, left and right centrifugal 1-to-2, left and right centripetal 2-to-1) and analyzed via rANOVA with the factors direction (left vs. right), orientation (centrifugal vs. centripetal), and proximity (proximal vs. distal). Only the EOG data, used for deriving saccade latency data in a corresponding experiment, will be reported here. The EEG data from this corresponding experiment will be reported elsewhere.

\section{RESULTS}

\section{BEHAVIORAL RESULTS fMRI STUDY}

The horizontal eye-position trace extracted from $24 \mathrm{~s}$ of a single subject's fMRI session is depicted in Figure 2A and includes all eight saccade types. The eye-position data of three of the subjects could not be analyzed due to technical problems. After rejecting an average of $10 \%$ of all trials due to blinks and other artifacts (e.g., eye-tracking signal disturbances caused by the fMRI gradients) in the remaining 13 data sets, the average saccade-task performance in the included trials was well above 95\% (rates of correctly executed saccades: centrifugal 0-to-1 $=0.97$; centripetal 1 -to-0 $=0.966$; centrifugal 1-to-2 =0.978; centripetal 2-to-1 =0.979). However, the general noise level and the restricted temporal resolution of $30 \mathrm{~Hz}$ did not allow for an accurate analysis of SRT differences in the scanner. The training prior to the scanning session, the online video monitoring, and the high percentage of correct trials indicated that the task was performed accurately. For a more precise analysis,

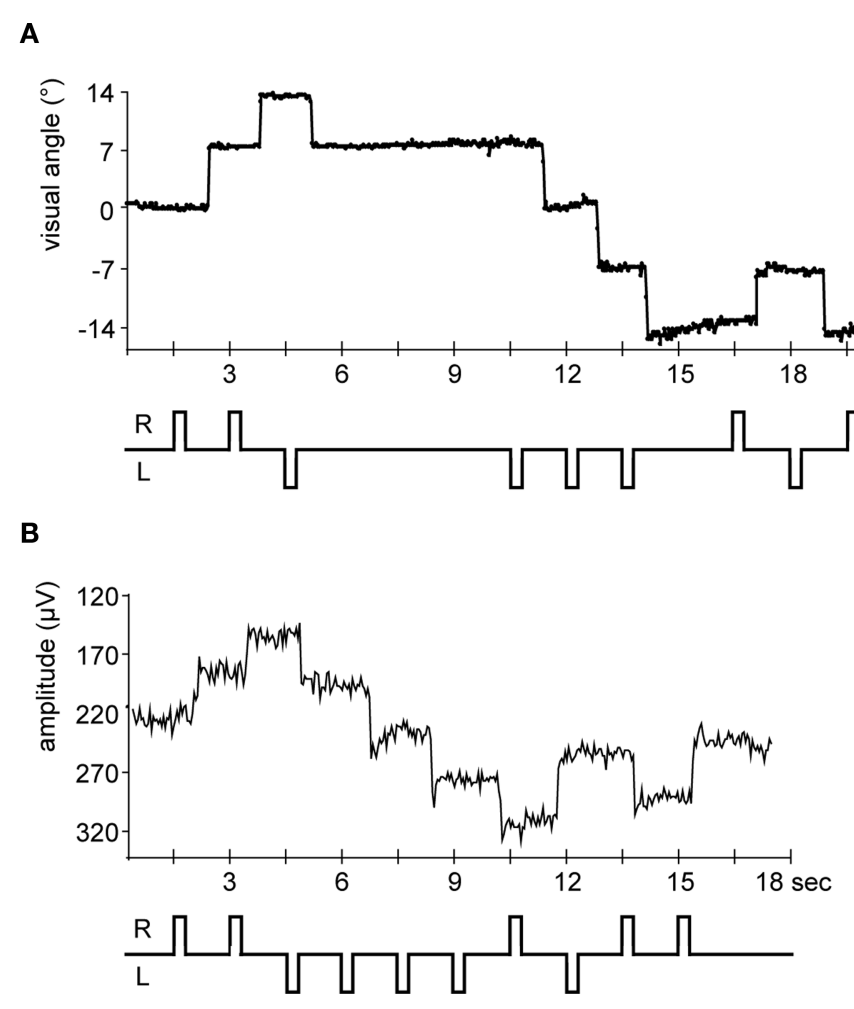

FIGURE 2 | Eye-position traces and saccadic reaction times (SRTs). (A) A single subject's horizontal eye-position trace from the $\mathrm{fMRI}$ scanning session is depicted for a sequence of $24 \mathrm{~s}$ (16 TRs) including saccades to all five possible positions at $0^{\circ}, \pm 7^{\circ}$, and $\pm 14^{\circ}$ of visual angle. According saccade direction is schematically depicted below, i.e., $R=$ rightward and $L=$ leftward. (B) The same trial sequence is shown as a single subject's horizontal EOG channel depicting rightward and leftward saccades, respectively (upward vs. downward

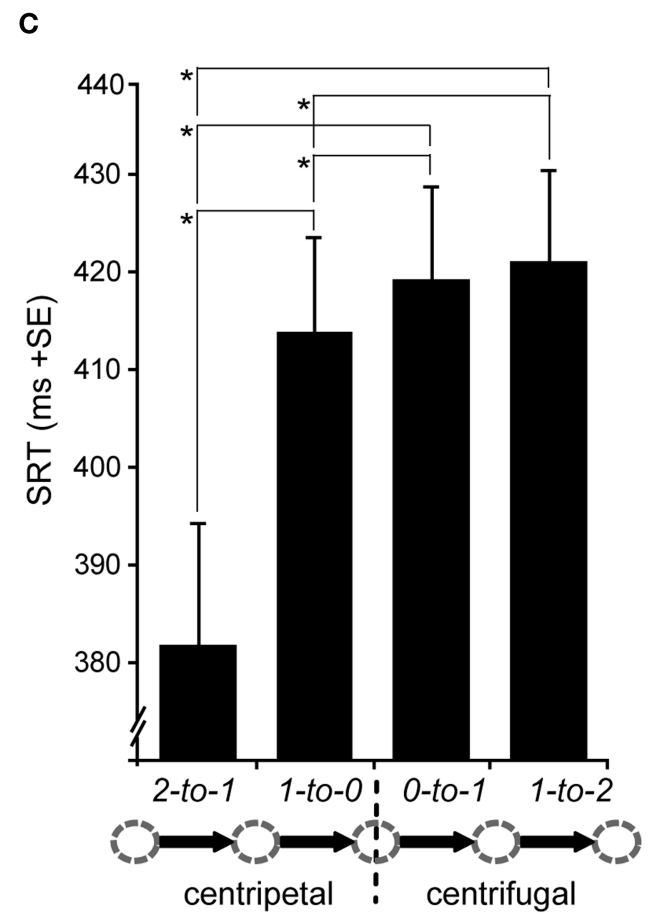

trace shifts). (C) SRTs extracted from the EOG data and averaged across leftward and rightward saccades for each condition (arrow legend exemplary depicts rightward shifts). SRTs were shorter for centripetal as compared to centrifugal saccades. Moreover, the SRT advantage for centripetal saccades increased with greater distance to the primary position. Error bars depict the standard error (SE) of the mean across subjects; asterisks indicate significant $T$-tests. 
particularly with respect to SRTs, the saccadic task performance in the equivalent EOG study, with much higher temporal resolution capability, was analyzed.

\section{BEHAVIORAL RESULTS EOG STUDY}

Figure 2B depicts a single subject's EOG trace of the identical trial sequence as the fMRI study (albeit with shorter SOAs). On average, no more than $6 \%$ of the trials in each condition had to be excluded due to artifacts in the EOG (e.g., blinks). Regarding the remaining trials, subjects consistently performed saccades in the cued direction as indicated by a mean percentage of correct saccades well above 95\% (rates of correctly executed saccades: centrifugal 0-to- $1=0.993$; centripetal 1-to-0 $=0.994$; centrifugal 1 -to- $2=0.989$; centripetal 2 -to-1 $=0.999$ ). Thus, the least number of errors was made during centripetal saccades starting from the most eccentric square, presumably reflecting the predictability of saccade direction at this position.

In regards to the SRTs, rightward saccades tended to be slightly faster than leftward ones, although this difference did not quite reach significance [direction $F_{(1,15)}=4.2, p=0.058$ ]. Regardless, in that there were also no significant interactions of any of the factors with left- vs. right saccade direction, the SRTs were collapsed across leftward and rightward saccades. Statistical analysis of the collapsed SRTs revealed shorter latencies for centripetal (proximal $413 \mathrm{~ms}$; distal $382 \mathrm{~ms}$ ) as compared to centrifugal (proximal $419 \mathrm{~ms}$; distal $421 \mathrm{~ms}$ ) saccades as indicated by a main effect of orientation $\left[F_{(1,15)}=47.03, p<0.0001\right]$. Moreover, there was a main effect of proximity $\left[F_{(1,15)}=15.22, p=0.001\right]$ which was accompanied by a significant interaction of orientation by proximity $\left[F_{(1,15)}=28, p<0.0001\right]$, resulting from the SRT advantage for centripetal saccades increasing with greater distance from the primary position (Figure 2C). Post hoc T-tests revealed that centripetal saccades from the most eccentric position yielded faster SRTs as compared to all other saccades (all $p$-values $<0.0001$ ), again most likely reflecting the high directional predictability. Furthermore, centripetal saccades toward primary position (re-centering saccades) yielded shorter SRTs as compared to centrifugal saccades (both proximal and distal conditions: $p<0.01$ ). No significant SRT difference was observed between proximal and distal centrifugal saccades $(p=0.379)$.

\section{VOXEL-WISE fMRI ACTIVATIONS DURING SACCADE EXECUTION}

Figure 3A displays representative slices of the comparison between all saccade trials and fixation trials, a contrast that subtracts out the sensory processing of the stimulus change at the foveated location. The voxel-wise contrast revealed a network of regions involved in the execution of endogenously cued saccades that were located along the horizontal meridian, including large portions of bilateral primary visual cortex along the calcarine fissure, lateral occipital cortex, FEF, the supplementary eye field (SEF), IPS, and SC (a detailed description of activation clusters is provided in Table 1; $T$-values refer to the local activity maxima). Note that the displayed activation clusters are all based on the modified HRF model peaking at $4.5 \mathrm{~s}$, which was more effective for the BOLD signal within the SC (see also Wall et al., 2009). For comparison, an analogous voxel-wise analysis based on the standard HRF peaking at $6 \mathrm{~s}$ can be found in Table 2 .

\section{SIGNAL TIME COURSE WITHIN SELECTED ROIS}

In order to estimate the actual shape of the BOLD signal, the time course was extracted from the selected ROIs based on a shape-assumption-free FIR model. The extracted values averaged across all saccade conditions and hemispheres for the selected ROIs are displayed in Figure 3B. The BOLD response within all three areas extracted from this shape-assumption-free FIR model peaked at $4.5 \mathrm{~s}$, in accordance with our use of the non-standard canonical HRF.

\section{ROI-BASED ANALYSIS OF PARAMETER ESTIMATES}

In order to investigate effects of the three main factors (contralateral predominance, orientation, and proximity) in an orthogonal fashion, parameter estimates were extracted from the conditionindependent-selected ROIs (i.e., all saccade trials vs. fixation trials; Figure 3A) and analyzed via rANOVA. The analyses confirmed the contralateral predominance across all saccade conditions within the selected bilateral-pair ROIs (bilateral SC, FEF, and IPS; Figure 3C). The activity enhancement in the hemisphere contralateral to saccade direction was most robust within the IPS (main effect of contralateral predominance: $\left.F_{(1,15)}=56.8, p<0.0001\right)$, followed by the SC $\left[F_{(1,15)}=15.7, p=0.001\right]$ and the FEF $\left[F_{(1,15)}=5.1, p=0.039\right]$.

With regard to the saccade orientation and proximity relative to the primary position, we observed a main effect of orientation within the SC $\left[F_{(1,15)}=7.3, p=0.016\right]$, confirming that centrifugal saccades were associated with enhanced collicular activity as compared to centripetal saccades (Figure 4). In the cortical ROIs, however, no main effects of saccade orientation or proximity to primary position were observed. However, the contralateral enhancement in IPS was significantly pronounced during centrifugal saccades [interaction of contralateral predominance $\times$ orientation: $\left.F_{(1,15)}=7.4, p=0.016\right]$. In addition, IPS displayed a tendency for a greater contralateral enhancement with increasing distance to the primary position, as indicated by a trend in the interaction term [contralateral predominance $\times$ proximity: $F_{(1,15)}=4.19, p=0.059$ ]. An analogous analysis within the FEF revealed a significant interaction between orientation and proximity $\left[F_{(1,15)}=5.9, p=0.028\right]$. This interaction reflected an inversion of activity differences between centripetal and centrifugal saccades dependent on the proximity to the primary position (i.e., proximal: centripetal > centrifugal; distal: centripetal $<$ centrifugal). No additional main or interaction effects were observed in the ROI analyses (all $p$-values $>0.1$ ).

Post hoc specific comparisons using two-tailed paired $T$-tests were performed focusing on activity within the predominant SC (i.e., contralateral to saccade direction) to further investigate the observed differences due to orientation and proximity within the respective predominant hemisphere. Activity in the predominant $\mathrm{SC}$ was significantly higher during distal centrifugal saccades (i.e., 1-to-2) than during both proximal (1-to-0) and distal (2-to-1) centripetal saccades (proximal $T_{(15)}=3.08, p=0.008$; distal $T_{(15)}=2.24$, $p=0.04$ ). Furthermore, centripetal saccades from the most eccentric position were associated with diminished SC activity as compared to centrifugal saccades from primary position $\left[T_{(15)}=3.99\right.$, $p=0.001]$.

Regarding activity in the predominant hemispheres of FEF and IPS, the only significant difference observed was between distal centrifugal (1-to-2) and distal centripetal saccades (2-to-1), with the 


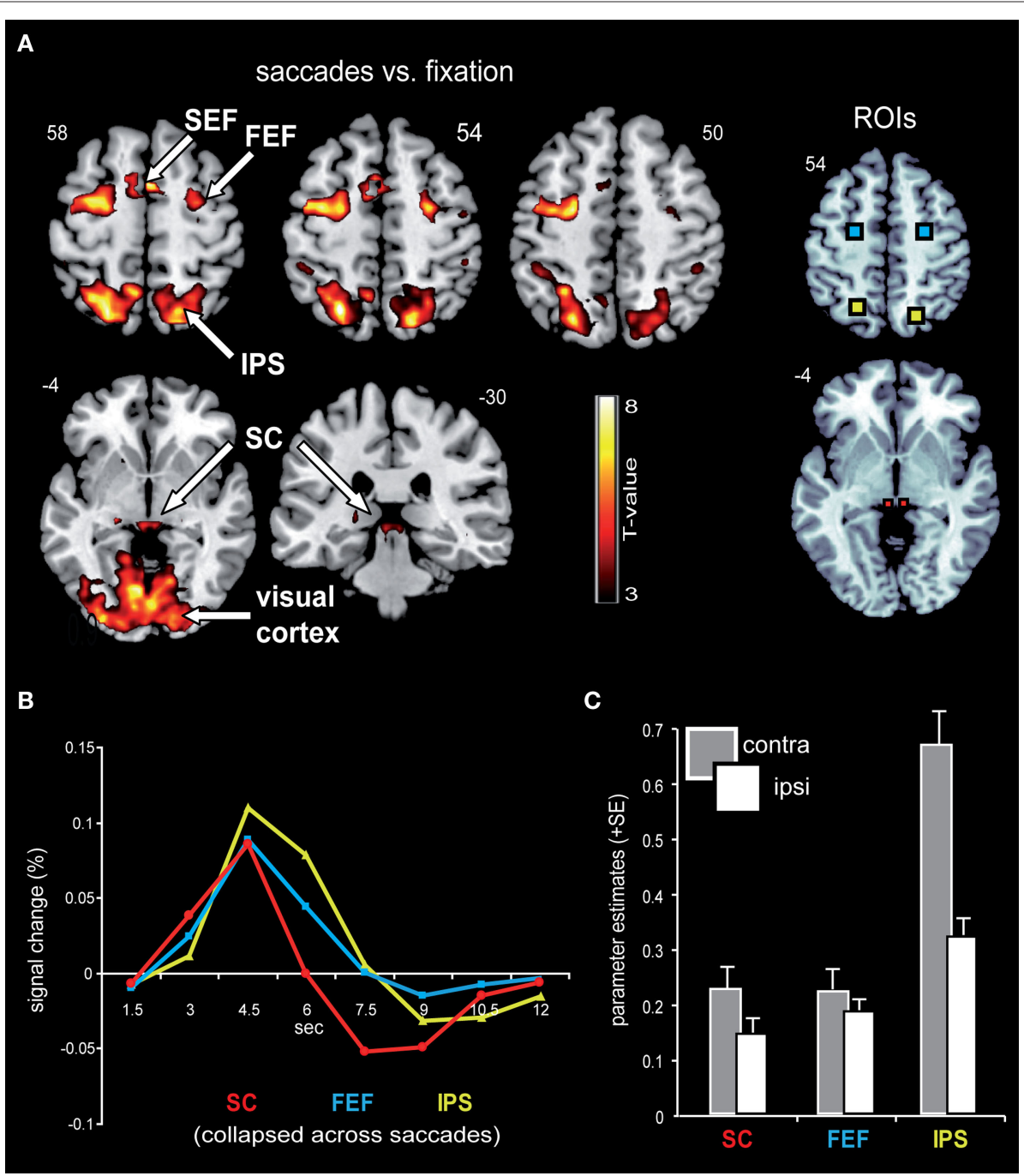

FIGURE 3 | Saccade-related BOLD activity. (A) Saccade trials, as compared to fixation trials, were associated with robust activity in a broad network that included bilateral IPS, FEF, SEF, SC, as well as large parts of the visual cortex. This contrast was based on an HRF that has been optimized for subcortical activity, with a peak at $4.5 \mathrm{~s}$. Activity is displayed on a standardT1-weighted image (display cut-off $T=3$; extent threshold $k=15$ ). (B) Mean BOLD time courses for the selected ROIs, namely the SC (depicted in red), FEF (blue), and IPS (yellow) are displayed averaged across all saccade trials and both hemispheres. (C) The collapsed ROI-based parameter estimates (beta) for leftward and rightward saccades with respect to the hemisphere contra- and ipsilateral to saccade direction revealed a relative activity enhancement in the contralateral (gray bars) as compared to the ipsilateral (white bars) hemisphere within SC, FEF, and IPS. former eliciting higher activity in the predominant FEF $\left[T_{(15)}=2.68\right.$, $p=0.017]$. However, there was a tendency for higher activity within the predominant FEF for proximal centrifugal saccades starting from primary position ( 0 -to- 1$)$ as compared to distal centripetal saccades [2-to-1; $\left.T_{(15)}=2, p=0.064\right]$. No further post hoc $T$-tests reached significance (all $p$-values $>0.1$ ). An additional ROI analysis within the mPFC bilaterally that was conducted to investigate differential default-mode network activity during the different saccade types revealed a significant effect of orientation $\left[F_{(1,15)}=10.49, p=0.006\right]$, with increased deactivation levels for centrifugal as compared to centripetal saccades. Post hoc specific $T$-tests verified that this main effect was mainly driven by significant differences between distal centrifugal (1-to-2) and proximal centripetal (1-to-0) saccades $\left[T_{(15)}=2.39, p=0.030\right]$ and between proximal centrifugal (0-to- 1$)$ and distal centripetal (2-to-1) saccades $\left[T_{(15)}=3.37, p=0.004\right]$.
It is important to note that the high directional predictability of distal centripetal saccades (2-to-1) imposes limitations on their use for direct comparisons with the remaining saccade types. In contrast, proximal centripetal (1-to-0) and distal centrifugal (1-to-2) saccades were closely matched regarding shift amplitude, directional probability, and the starting gaze-deviation from primary position, thus yielding the "purest" comparison regarding the investigation of the re-centering bias.

In summary, activity within the SC, as well as in FEF and IPS, was enhanced contralateral to saccade direction (contralateral predominance, see Figure $3 \mathrm{C}$ ). When focusing on the predominant hemisphere, activity within the SC was diminished during centripetal as compared to centrifugal saccades (Figure 4). This was especially true for saccades starting from the most eccentric position, but the effect was still present for the more proximal 
Table 1 | Activity clusters for all saccades vs. fixation (non-standard HRF).

\begin{tabular}{|c|c|c|c|c|c|}
\hline \multirow[b]{2}{*}{ Region } & \multirow[b]{2}{*}{ L/R } & \multicolumn{3}{|c|}{ MNI coordinates } & \multirow[b]{2}{*}{$T$-value } \\
\hline & & $x$ & $y$ & $z$ & \\
\hline \multicolumn{6}{|l|}{ SACCADES > FIXATION } \\
\hline Anterior lingual gyrus & $\mathrm{L}$ & -10 & -66 & 2 & 11.94 \\
\hline Posterior lingual gyrus & $\mathrm{R}$ & 16 & -90 & -10 & 11.10 \\
\hline Middle occipital gyrus & $\mathrm{L}$ & -30 & -90 & -6 & 10.35 \\
\hline IPS & $\mathrm{L}$ & -24 & -64 & 54 & 9.64 \\
\hline Posterior lingual gyrus & $\mathrm{R}$ & 6 & -82 & 0 & 9.44 \\
\hline BA30 & $\mathrm{R}$ & 12 & -64 & 10 & 8.83 \\
\hline Cuneus & $\mathrm{L}$ & -12 & -80 & 2 & 8.63 \\
\hline Cuneus & $\mathrm{R}$ & 12 & -82 & 2 & 8.62 \\
\hline SEF & $\mathrm{R}$ & 2 & 4 & 58 & 8.28 \\
\hline FEF & $\mathrm{L}$ & -24 & -6 & 50 & 7.93 \\
\hline Posterior occipital cortex & $\mathrm{R}$ & 2 & -88 & 2 & 7.92 \\
\hline IPS & $\mathrm{L}$ & -32 & -60 & 60 & 7.74 \\
\hline IPS & $\mathrm{R}$ & 18 & -66 & 56 & 7.57 \\
\hline Lateral FEF & $\mathrm{L}$ & -40 & -4 & 54 & 7.08 \\
\hline FEF & $\mathrm{R}$ & 26 & -6 & 54 & 7.07 \\
\hline SEF & $\mathrm{L}$ & -8 & 0 & 56 & 6.58 \\
\hline Posterior occipital cortex & $\mathrm{L}$ & -14 & -90 & -10 & 6.49 \\
\hline SC & $\mathrm{L}$ & -4 & -28 & -6 & 4.74 \\
\hline Anterior IPS & $\mathrm{L}$ & -42 & -42 & 52 & 4.49 \\
\hline SC & $\mathrm{R}$ & 4 & -28 & -4 & 4.40 \\
\hline Anterior IPS & $\mathrm{R}$ & 48 & -38 & 52 & 4.09 \\
\hline Lateral geniculate nucleus & $\mathrm{L}$ & -22 & -24 & -4 & $5.49^{\mathrm{a}}$ \\
\hline Inferior parietal lobe & $\mathrm{R}$ & 54 & -44 & 20 & $4.42^{\mathrm{a}}$ \\
\hline Inferior parietal lobe & $L$ & -60 & -42 & 24 & $4.00^{\mathrm{a}}$ \\
\hline Pulvinar & $\mathrm{L}$ & -22 & -30 & 4 & $3.77^{\mathrm{a}}$ \\
\hline Thalamus & $\mathrm{L}$ & -10 & -18 & 10 & $3.64^{a}$ \\
\hline Putamen & $\mathrm{R}$ & 24 & 8 & -8 & $3.28^{a}$ \\
\hline
\end{tabular}

Cluster maxima are based on the non-standard HRF model peaking at $4.5 \mathrm{~s}$.

T-value: thresholded at $p=0.001$ ('thresholded at $p=0.005$ ), extent threshold $k=15$.

$L$, left hemisphere; $R$, right hemisphere; MNI, Montreal Neurological Institute.

ones. Importantly, this re-centering activity modulation in the SC closely matched the pattern of the corresponding SRTs (Figure 2C). At the cortical level, the only robust orientation effect within paired comparisons was the increased activity in the predominant FEF for distal centrifugal saccades (1-to-2) as compared to centripetal saccades starting at the most eccentric position (2-to-1).

\section{DISCUSSION}

\section{BEHAVIORAL PERFORMANCE}

The general performance level in the saccade task was well above 95\% in both the fMRI study and the analogous EOG one. Based on the highly comparable performance, we based the SRT analysis on the EOG data, due to its considerably higher temporal resolution, as well as the higher trial numbers and superior data quality. Mean SRTs varied between 380 and 420 ms depending on the condition, consistent with the normal SRT range of centrally cued saccades (Walker et al., 2000).
Table 2 | Activity clusters for all saccades vs. fixation (standard HRF).

\begin{tabular}{|c|c|c|c|c|c|}
\hline \multirow[b]{2}{*}{ Region } & \multirow[b]{2}{*}{ L/R } & \multicolumn{3}{|c|}{ MNI coordinates } & \multirow[b]{2}{*}{$T$-value } \\
\hline & & $x$ & $y$ & $z$ & \\
\hline \multicolumn{6}{|l|}{ SACCADES > FIXATION } \\
\hline FEF & $\mathrm{L}$ & -32 & -4 & 56 & 8.04 \\
\hline Lateral FEF & $\mathrm{L}$ & -42 & -4 & 54 & 7.93 \\
\hline IPS & $L$ & -30 & -58 & 56 & 7.91 \\
\hline IPS & $\mathrm{R}$ & 20 & -68 & 56 & 7.12 \\
\hline Inferior occipital gyrus & $\mathrm{L}$ & -26 & -96 & -6 & 6.39 \\
\hline Middle occipital gyrus & $\mathrm{L}$ & -32 & -92 & 0 & 5.96 \\
\hline Cuneus & $\mathrm{R}$ & 12 & -74 & 14 & 5.95 \\
\hline Posterior lingual gyrus & $\mathrm{L}$ & -16 & -94 & -10 & 5.70 \\
\hline Anterior occipital cortex (BA18) & $L$ & -14 & -72 & 14 & 5.36 \\
\hline Inferior occipital gyrus & $\mathrm{R}$ & 28 & -92 & -8 & 5.20 \\
\hline FEF & $\mathrm{R}$ & 24 & -6 & 54 & 4.98 \\
\hline Cuneus & $L$ & -12 & -68 & 6 & 4.79 \\
\hline Posterior lingual gyrus & $\mathrm{R}$ & 16 & -96 & -8 & 4.29 \\
\hline Middle occipital gyrus & $L$ & -44 & -74 & -8 & $5.17^{a}$ \\
\hline Putamen & $\mathrm{L}$ & -26 & 6 & 6 & $4.13^{\mathrm{a}}$ \\
\hline
\end{tabular}

Cluster maxima are based on the non-standard HRF model peaking at $4.5 \mathrm{~s}$.

T-value: thresholded at $p=0.001$ (athresholded at $p=0.005$ ), extent threshold $k=15$.

$L$, left hemisphere; $R$, right hemisphere; MNI, Montreal Neurological Institute.

In line with the notion of a facilitation for saccades toward the primary position (re-centering bias), we observed shorter SRTs for centripetal saccades, as has previously been reported in both animals (Albano and Wurtz, 1982; McIlwain, 1986; Pare and Munoz, 1996) and humans (Laurutis and Robinson, 1986; Fuller, 1996). This latency bias has been initially associated with the general dynamics of eye-head coordination in natural head-free gaze shifts (combined eye- and head-movement), but appears to be also conserved in a headrestrained setting. Importantly, the re-centering bias was observed not only for the saccades starting from the most eccentric positions, which differ in their directional predictability from all other saccades, but also when comparing centripetal and centrifugal saccades starting at position "1" (1-to-0 vs. 1-to-2; see Figure 1C). This latter comparison is considerably more compelling since these saccade types differ only in their centripetal (i.e., re-centering) vs. centrifugal orientation relative to the primary position while being matched regarding both starting position and directional predictability.

\section{SACCADE-RELATED ACTIVITY AND CONTRALATERALITY}

The comparison of all saccades to fixation trials provided a general activity map for saccade generation, including the primary areas of interest in this study - namely, the SC, IPS, and FEF. We also observed activity clusters in various regions of visual cortex, as well as in some additional subcortical regions related to stimulus and saccade processing (including medial thalamus, lateral geniculate nucleus, pulvinar, and putamen). Although several of these areas are implicated in saccade processing (e.g., primary visual cortex, see Bodis-Wollner et al., 1997; Sylvester et al., 2005; Rieger et al., 2008), we focused on the two main cortical areas (i.e., FEF and IPS) and the key subcortical area (i.e., SC) that are central to the generation of saccadic eye movements. 


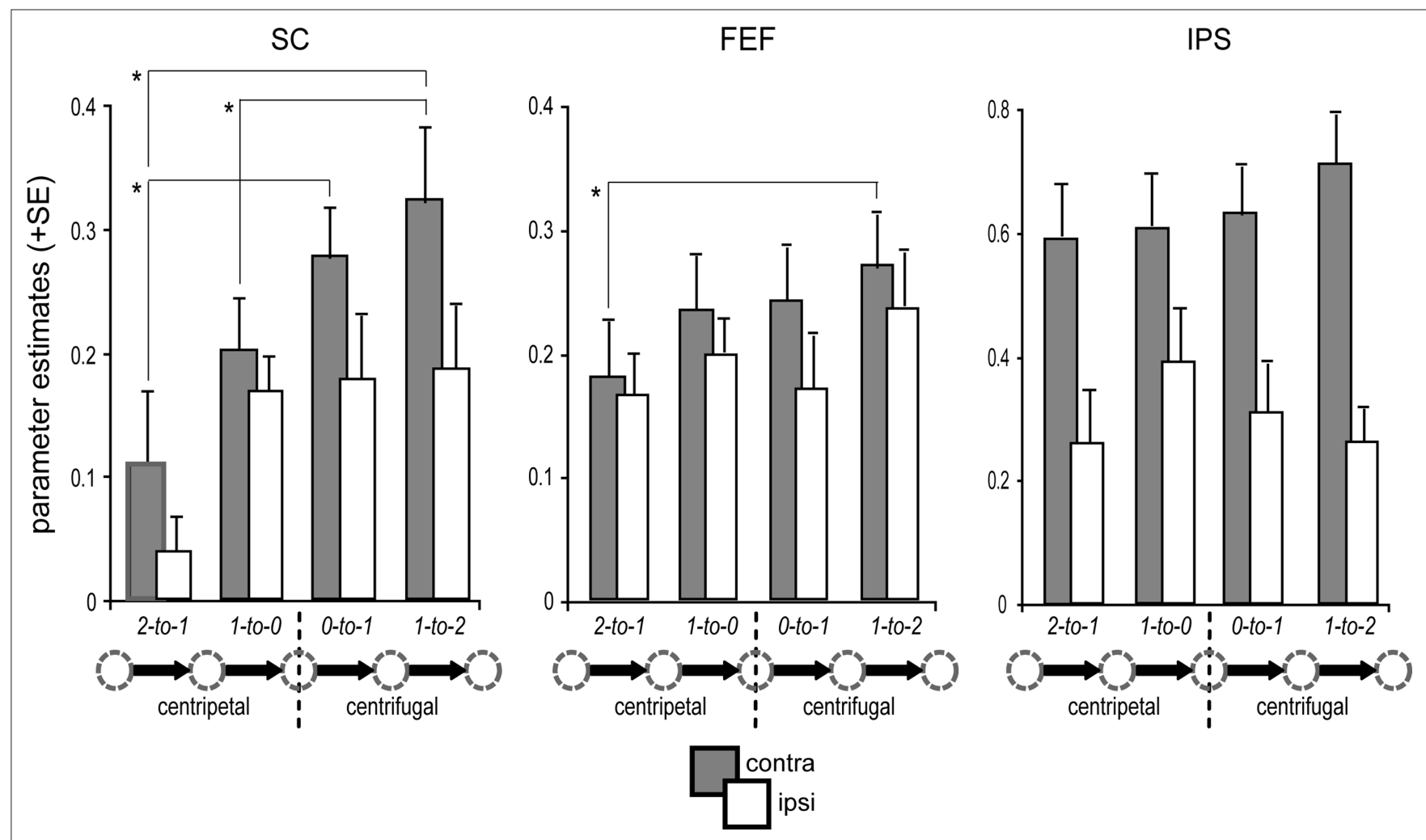

FIGURE 4 | Condition-specific BOLD response. ROI-based parameter estimates are collapsed for leftward and rightward saccades with respect to the hemisphere contralateral (gray bars) and ipsilateral (white bars) to saccade direction. Activity in the predominant SC (i.e., contralateral) was modulated by the orientation of the saccade relative to primary position. In particular, the generation of re-centering saccades (1-to-0) was associated with significantly decreased activity as compared to centrifugal saccades (1-to-2) of identical amplitude, directional probability, and equivalent starting position (arrow legend exemplary depicts rightward shifts). Activity in the contralateral FEF and IPS was not significantly modulated by the saccade orientation relative to primary position. Error bars depict the standard error (SE) of the activity means across subjects; asterisks indicate significant T-tests focusing on contralateral activity only.
The ROI-based analysis of the BOLD activity revealed that activity in the SC was significantly enhanced contralateral to the direction of the saccade target, a pattern that has been demonstrated in numerous studies in animals (e.g., Goldberg and Wurtz, 1972; Field et al., 2008) and recently in humans (Krebs et al., 2010). A contralateral bias was also observed in the cortical eye fields (FEF and IPS), matching the results of previous fMRI studies using memory-guided saccade paradigms (Schluppeck et al., 2005; Curtis and Connolly, 2008). Importantly, the contralateral predominance appeared to be independent of the additional experimental factors, i.e., orientation and proximity relative to the primary position, except for a relative increase in contralateral IPS activity during centrifugal as compared to centripetal saccades, which might be due to overlapping topographic representations of left and right IPS (Kastner et al., 2007; Konen and Kastner, 2008).

It is important to consider whether the observed contralateral activity enhancement in saccade-related regions could have been simply induced by differential sensory input. First, configurational aspects argue against this possibility. For centrifugal saccades, the majority of both the pre- and post-saccadic visual input from the stimulus array would lie in the visual field contralateral to the saccade direction, which would thus tend to lead to enhanced activity in the ipsilateral hemisphere. For centripetal saccades, on the other hand, the majority of pre- and post-saccadic visual input would lie in the visual field ipsilateral to the saccade direction, and thus a robust contribution of the visual input should tend to produce a relatively increased contralateral predominance for centripetal saccades close to the primary position. None of these asymmetries was observed in the present data. Furthermore, a recent study comparing a covert and overt version of a spatial attention task found highly similar activity levels in the cortical eye fields, despite the fact that only overt trials entailed a change in eye position and thus a change of the current visual input (Ikkai and Curtis, 2008).

\section{DIFFERENTIAL ACTIVITY DURING RE-CENTERING SACCADES}

In keeping with the behavioral analysis, the best controlled comparison for investigating the underlying neural pattern of the recentering bias is the comparison of centripetal and centrifugal saccades starting at the same orbital position (1-to-0 vs. 1-to-2). Our results indeed show that activity within the predominant SC side (contralateral to saccade direction) was significantly diminished during centripetal re-centering saccades as compared to centrifugal saccades from the equivalent starting position (Figure 4), a pattern that was paralleled by the SRT differences (Figure 2C).

Our observation of reduced evoked SC activity in humans during re-centering as compared to centrifugal saccades is consistent with the animals studies described above, and might be related to dynamic gain field modulations in collicular movement neurons 
(Van Opstal et al., 1995; Salinas and Thier, 2000). In particular, the firing activity of a large portion of collicular neurons are modulated by the orbital position (Van Opstal et al., 1995; Pare and Munoz, 2001; Campos et al., 2006), and the SC thus appears to be in the position to effectively integrate eye- and head-position information (Sparks, 2002; Kardamakis and Moschovakis, 2009). It has been proposed that for eye positions that deviate from the primary position, stronger muscular elastic forces need to be overcome to initiate a saccade to an even more centrifugal position as compared to a centripetal saccade. For example, the initiation of rightward saccades with identical amplitude would require differential levels of activity in the left SC (contralateral to saccade direction) depending on the starting point relative to the primary position (see Figure 1C). Alternatively, or in addition, there might be an involvement of specific neurons in the posterior SC that have been shown to re-center the eyes independently of the initial position (Guitton et al., 1980), a mechanism that could result in less requirements for movement neurons that code a specific saccade vector. A recent study has shown evidence for a preferential coding of the primary position in early visual cortex (V1) of monkeys (Durand et al., 2010). Therefore it seems likely that the re-centering bias relies on projections between visual cortex and the SC (Fries, 1984), which is consistent with a recent study reporting egocentric referenceframe properties of human visual cortex using retinotopic mapping (Rieger et al., 2008).

While the SC was clearly sensitive to the orientation of the saccade vector relative to the primary position, neither of the cortical eye fields (i.e., FEF and IPS) was systematically influenced by this factor. Although the IPS is generally involved in the continuous updating of visual space by transforming coordinates of the eye- and head-centered reference frames (Duhamel et al., 1992; Colby et al., 1995; Medendorp et al., 2003), the present data reveals no re-centering-specific activity modulation in this region, which is consistent with earlier findings (Rieger et al., 2008). Regarding the FEF, it has been reported that, under some conditions, different orbital starting positions change the resulting saccade vector elicited by micro-stimulation in humans (Blanke and Seeck, 2003). However, these modulations were restricted to differences in starting positions along the vertical meridian while variable starting positions along the horizontal meridian did not influence direction or amplitude of the elicited saccades.

It is well established that activity in the cortical eye fields is generally increased during tasks requiring higher levels of top-down processing both in overt and covert attention paradigms in humans (reviewed in Corbetta and Shulman, 2002). More specifically, activity in both IPS and FEF is increased during the execution of endogenously cued (voluntary) saccades, as compared to exogenously cued (reflexive) saccades, an effect that has been interpreted as likely reflecting the higher processing demands during cue interpretation and saccade preparation for the former (Mort et al., 2003). Since

\section{REFERENCES}

Albano, J. E., and Wurtz, R. H. (1982). Deficits in eye position following ablation of monkey superior colliculus, pretectum, and posterior-medial thalamus. J. Neurophysiol. 48, 318-337.
Ashburner, J., and Friston, K. J. (1999). Nonlinear spatial normalization using basis functions. Hum. Brain Mapp. 7, 254-266.

Astafiev, S. V., Shulman, G. L., Stanley, C. M., Snyder, A. Z., Van Essen, D. C., and

the current paradigm required the voluntary initiation of saccades following central instructional cues, we expected a similar degree of engagement of these regions irrespective of saccade orientation. The lack of significant cortical activity changes during re-centering saccades is thus consistent with the view that the re-centering bias is a fairly automatized process subsequent to cue interpretation and target selection and is thus mainly implemented at the subcortical level, where current eye- and head-positions as well as the future gaze position are integrated (e.g., Campos et al., 2006).

It remains possible, however, that potential cortical contributions to the re-centering bias may have been diluted by neural processes that are common to all saccade types, i.e., cue interpretation and target selection, which can not be easily separated due to the low temporal resolution of the BOLD signal. Such a possibility might explain why there appeared to be a trend in both FEF and IPS for reduced activity during 1-to- 0 as compared to 1 -to- 2 saccades (see Figure 4) which did not reach significance. Along similar lines, it is furthermore possible that this trend might have arisen from a corollary signal from SC to these cortical regions (Sommer and Wurtz, 2004, 2008) conveying a copy of the re-centering-related motor signal.

While no significant activity modulation was found in cortical areas that are directly implicated in saccade processing, the present data still provide some evidence for subcortical-cortical interactions. In particular, and consistent with our previous work (Krebs et al., 2010) the MPFC, a region associated with the defaultmode network (Gusnard et al., 2001; Raichle et al., 2001), exhibited differential activity during centrifugal and centripetal saccades with considerably more pronounced deactivation for the former. Based on the notion that less demanding tasks are associated with reduced deactivations in this area, this pattern might be related to the diminished neural activity that we observed in the SC during the execution of re-centering saccades.

\section{CONCLUSIONS}

The current study investigated the neural basis of the re-centering bias in humans that has been demonstrated behaviorally. We systematically varied saccade direction and orbital starting position while keeping saccade amplitude and directional predictability constant across the conditions of interest. The observed reduced $\mathrm{BOLD}$ response in the SC during re-centering saccades is in line with the notion that this subcortical brain region integrates information about future gaze positions as well as eye- and head-position signals, thus engendering effective exploration of the visual environment.

\section{ACKNOWLEDGMENTS}

We thank Ken Roberts and Michael Scholz for technical support. This work was funded by NIH grants R01-MH060415 and R01NS051048 to Marty G. Woldorff.

Corbetta,M. (2003).Functional organization of human intraparietal and frontal cortex for attending, looking, and pointing. J. Neurosci. 23, 4689-4699.

Blanke, O., and Seeck, M. (2003). Direction of saccadic and smooth eye movements induced by electrical stimulation of the human frontal eye field: effect of orbital position. Exp. Brain Res. 150, 174-183.

Blanke, O., Spinelli, L., Thut, G., Michel, C. M., Perrig, S., Landis, T., and Seeck, 
M. (2000). Location of the human frontal eye field as defined by electrical cortical stimulation: anatomical, functional and electrophysiological characteristics. Neuroreport 11, 1907-1913.

Blatt, G. J., Andersen, R. A., and Stoner, G. R. (1990).Visual receptive field organization and cortico-cortical connections of the lateral intraparietal area (area LIP) in the macaque. J. Comp. Neurol. 299, 421-445.

Bodis-Wollner, I., Bucher, S. F., Seelos, K. C., Paulus, W., Reiser, M., and Oertel, W. H. (1997). Functional MRI mapping of occipital and frontal cortical activity during voluntary and imagined saccades. Neurology 49, 416-420.

Boehnke, S. E., and Munoz, D. P. (2008). On the importance of the transient visual response in the superior colliculus. Curr. Opin. Neurobiol. 18, 544-551.

Brett, M., Anton, J.-L., Valabregue, R., and Poline, J.-P. (2002). Region of interest analysis using an SPM toolbox (abstract). Neuroimage 16 (available on CD-Rom).

Bruce, C. J., and Goldberg, M. E. (1985). Primate frontal eye fields. I. Single neurons discharging before saccades. J. Neurophysiol. 53, 603-635.

Campos, M., Cherian, A., and Segraves, M. A. (2006). Effects of eye position upon activity of neurons in macaque superior colliculus. J. Neurophysiol.95, 505-526.

Colby, C.L., Duhamel, J. R., and Goldberg, M. E. (1995). Oculocentric spatial representation in parietal cortex. Cereb. Cortex 5, 470-481.

Corbetta, M., and Shulman, G. L. (2002). Control of goal-directed and stimulusdriven attention in the brain. Nat. Rev. Neurosci. 3, 201-215.

Crapse, T. B., and Sommer, M. A. (2009). Frontal eye field neurons with spatial representations predicted by their subcortical input. J. Neurosci. 29, 5308-5318.

Curtis, C. E., and Connolly, J. D. (2008). Saccade preparation signals in the human frontal and parietal cortices. J. Neurophysiol. 99, 133-145.

DuBois, R. M., and Cohen, M. S. (2000). Spatiotopic organization in human superior colliculus observed with fMRI. Neuroimage 12, 63-70.

Duhamel, J.R., Colby, C. L., and Goldberg, M.E. (1992). The updating of the representation of visual space in parietal cortex by intended eye movements. Science 255, 90-92.

Durand, J. B., Trotter, Y., and Celebrini, S. (2010). Privileged processing of the straight-ahead direction in primate area V1. Neuron 66, 126-137.
Fecteau, J. H., and Munoz, D. P. (2006). Salience, relevance, and firing: a priority map for target selection. Trends Cogn. Sci. 10, 382-390.

Field, C. B., Johnston, K., Gati, J. S., Menon, R. S., and Everling, S. (2008). Connectivity of the primate superior colliculus mapped by concurrent microstimulation and event-related FMRI. PLOS ONE 3, e3928. doi: 10.1371/journal.pone.0003928.

Fries, W. (1984). Cortical projections to the superior colliculus in the macaque monkey: a retrograde study using horseradish peroxidase. J. Comp. Neurol. 230, 55-76.

Friston, K., Holmes, A. P., Worsley, K. J., Poline, J. B., Frith, C. D., and Frackowiak, R. S. (1995). Statistical parametric maps in functional imaging: a general linear approach. Hum. Brain Mapp. 2, 189-210.

Fuller, J. H. (1996). Eye position and target amplitude effects on human visual saccadic latencies. Exp. Brain Res. 109, 457-466.

Gaymard, B., Ploner, C. J., Rivaud, S., Vermersch, A. I., and PierrotDeseilligny, C. (1998). Cortical control of saccades. Exp. Brain Res. 123, 159-163.

Goldberg, M.E., and Wurtz, R. H. (1972). Activity of superior colliculus in behaving monkey. II. Effect of attention on neuronal responses. J. Neurophysiol. 35, 560-574.

Guitton, D., Crommelinck, M., and Roucoux, A. (1980). Stimulation of the superior colliculus in the alert cat. I. Eye movements and neck EMG activity evoked when the head is restrained. Exp. Brain Res. 39, 63-73.

Gusnard, D. A., Raichle, M. E., and Raichle, M. E. (2001). Searching for a baseline: functional imaging and the resting human brain. Nat. Rev. Neurosci. 2, 685-694.

Hinrichs, H., Scholz, M., Tempelmann, C., Woldorff, M. G., Dale, A. M., and Heinze, H.-J. (2000). Deconvolution of event-related fMRI responses in fast-rate experimental designs: tracking amplitude variations. J. Cogn. Neurosci. 12, 76-89.

Ignashchenkova, A., Dicke, P. W. Haarmeier, T., and Thier, P. (2004). Neuron-specific contribution of the superior colliculus to overt and covert shifts of attention. Nat. Neurosci. 7, 56-64.

Ikkai,A., and Curtis, C.E. (2008). Cortical activity time locked to the shift and maintenance of spatial attention. Cereb. Cortex 18, 1384-1394.

Kardamakis,A.A., and Moschovakis, A. K. (2009). Optimal control of gaze shifts. J. Neurosci. 29, 7723-7730.
Kastner, S., DeSimone, K., Konen, C. S. Szczepanski, S. M., Weiner, K. S., and Schneider, K. A. (2007). Topographic maps in human frontal cortex revealed in memory-guided saccade and spatial working-memory tasks. $J$. Neurophysiol. 97, 3494-3507.

Konen, C. S., and Kastner, S. (2008). Representation of eye movements and stimulus motion in topographically organized areas of human posterior parietal cortex. J. Neurosci. 28 , 8361-8375.

Koyama, M., Hasegawa, I., Osada, T., Adachi, Y., Nakahara, K., and Miyashita, Y. (2004). Functional magnetic resonance imaging of macaque monkeys performing visually guided saccade tasks: comparison of cortical eye fields with humans. Neuron 41, 795-807.

Krebs, R. M., Woldorff, M. G., Tempelmann, C., Bodammer, N., Noesselt, T., Boehler, C. N., Scheich, H., Hopf, J. M., Duzel, E., Heinze, H. J., and Schoenfeld, M.A. (2010). Highfield FMRI reveals brain activation patterns underlying saccade execution in the human superior colliculus. PLoS ONE 5, e8691. doi: 10.1371/journal. pone.0008691.

Kustov, A. A., and Robinson, D. L. (1996). Shared neural control of attentional shifts and eye movements. Nature 384, 74-77.

Laurutis, V.P., and Robinson, D.A. (1986) The vestibulo-ocular reflex during human saccadic eye movements. J. Physiol. 373, 209-233.

Luna, B., Thulborn, K. R., Strojwas, M. H., McCurtain, B. J., Berman, R. A., Genovese, C. R., and Sweeney, J. A. (1998). Dorsal cortical regions subserving visually guided saccades in humans: an fMRI study. Cereb. Cortex 8, 40-47.

McIlwain, J. T. (1986). Effects of eye position on saccades evoked electrically from superior colliculus of alert cats. J. Neurophysiol. 55, 97-112.

Medendorp, W. P., Goltz, H. C., Vilis, T. and Crawford, J. D. (2003). Gazecentered updating of visual space in human parietal cortex. J. Neurosci. 23, 6209-6214.

Mort, D. J., Perry, R. J., Mannan, S. K. Hodgson, T. L., Anderson, E., Quest, R., McRobbie, D., McBride, A. Husain, M., and Kennard, C. (2003). Differential cortical activation during voluntary and reflexive saccades in man. Neuroimage 18, 231-246.

Moschovakis, A. K. (1996). The superior colliculus and eye movement control. Curr. Opin. Neurobiol. 6, 811-816.

Muri, R. M., Iba-Zizen, M. T., Derosier, C., Cabanis, E.A., and Pierrot-Deseilligny, C. (1996). Location of the human posterior eye field with functional magnetic resonance imaging. J. Neurol. Neurosurg. Psychiatr. 60, 445-448.

Ollinger, J.M., Corbetta, M., and Shulman, G. L. (2001). Separating processes within a trial in event-related functional MRI. Neuroimage 13, 218-229.

Pare, M., and Munoz, D. P. (1996). Saccadic reaction time in the monkey: advanced preparation of oculomotor programs is primarily responsible for express saccade occurrence. $J$. Neurophysiol. 76, 3666-3681.

Pare, M., and Munoz, D. P. (2001). Expression of a re-centering bias in saccade regulation by superior colliculus neurons. Exp. Brain Res. 137, 354-368.

Paus, T. (1996). Location and function of the human frontal eye-field: a selective review. Neuropsychologia 34, 475-483.

Platt, M. L., and Glimcher, P. W. (1997). Responses of intraparietal neurons to saccadic targets and visual distractors. J. Neurophysiol. 78, 1574-1589.

Raichle, M. E., MacLeod, A. M., Snyder, A. Z., Powers, W. J., Gusnard, D. A., and Shulman, G. L. (2001). A default mode of brain function. Proc. Natl. Acad. Sci. U.S.A. 98, 676-682.

Rieger, J. W., Schoenfeld, M. A., Heinze, H.-J., and Bodis-Wollner, I. (2008). Different spatial organizations of saccade related BOLD-activation in parietal and striate cortex. Brain Res. 1233, 89-97.

Robinson, D. A. (1972). Eye movements evoked by collicular stimulation in the alert monkey. Vision Res. 12, 1795-1808.

Salinas, E., and Thier, P. (2000). Gain modulation: a major computational principle of the central nervous system. Neuron 27, 15-21.

Schall, J. D. (1995). Neural basis of saccade target selection. Rev. Neurosci. 6, 63-85.

Schiller, P. H., and Stryker, M. (1972). Single-unit recording and stimulation in superior colliculus of the alert rhesus monkey. J. Neurophysiol. 35, 915-924.

Schiller, P. H., and Tehovnik, E. J. (2001). Look and see: how the brain moves your eyes about. Prog. Brain Res. 134, 127-142.

Schluppeck, D., Glimcher, P., and Heeger, D. J. (2005). Topographic organization for delayed saccades in human posterior parietal cortex. J. Neurophysiol. 94, 1372-1384.

Schneider, K. A., and Kastner, S. (2005). Visual responses of the human superior colliculus: a high-resolution functional magnetic resonance imaging study. J. Neurophysiol. 94, 2491-2503. 
Schneider, K. A., and Kastner, S. (2009). Effects of sustained spatial attention in the human lateral geniculate nucleus and superior colliculus. J. Neurosci. 29, 1784-1795.

Serences, J. T., and Yantis, S. (2007). Spatially selective representations of voluntary and stimulus-driven attentional priority in human occipital, parietal, and frontal cortex. Cereb. Cortex 17, 284-293.

Sereno, M. I., Pitzalis, S., and Martinez, A. (2001). Mapping of contralateral space in retinotopic coordinates by a parietal cortical area in humans. Science 294, 1350-1354.

Silver, M. A., Ress, D., and Heeger, D. J. (2005). Topographic maps of visual spatial attention in human parietal cortex. J. Neurophysiol. 94, 1358-1371.

Sommer, M.A., and Tehovnik, E. J. (1997). Reversible inactivation of macaque frontal eye field. Exp. Brain Res. 116, 229-249.

Sommer, M. A., and Wurtz, R. H. (2004). What the brain stem tells the frontal cortex. I. Oculomotor signals sent from superior colliculus to frontal eye field via mediodorsal thalamus. $J$. Neurophysiol. 91, 1381-1402.

Sommer, M. A., and Wurtz, R. H. (2008). Brain circuits for the internal monitoring of movements. Annu. Rev. Neurosci. 31, 317-338.

Sparks, D., Rohrer, W. H., and Zhang, Y. (2000). The role of the superior colliculus in saccade initiation: a study of express saccades and the gap effect. Vision Res. 40, 2763-2777.

Sparks, D. L. (2002). The brainstem control of saccadic eye movements. Nat. Rev. Neurosci. 3, 952-964.

Sylvester, R., Haynes, J. D., and Rees, G. (2005). Saccades differentially modulate human LGN and V1 responses in the presence and absence of visual stimulation. Curr. Biol. 15, 37-41.

Tatler, B. W. (2007). The central fixation bias in scene viewing: selecting an optimal viewing position independently of motor biases and image feature distributions. J. Vision 7, 1-17.

Tehovnik, E. J., and Slocum, W. M. (2000). Effects of training on saccadic eye movements elicited electrically from the frontal cortex of monkeys. Brain Res. 877, 101-106.

Van Opstal, A. J., Hepp, K., Suzuki, Y., and Henn, V. (1995). Influence of eye position on activity in monkey superior colliculus. J. Neurophysiol. 74, 1593-1610.

Vitu, F., Kapoula, Z., Lancelin, D., and Lavigne, F. (2004). Eye movements in reading isolated words: evidence for strong biases towards the center of the screen. Vision Res. 44, 321-338.

Walker, R., Walker, D. G., Husain, M., and Kennard, C. (2000). Control of voluntary and reflexive saccades. Exp. Brain Res. 130, 540-544.

Wall, M. B., Walker, R., and Smith, A. T. (2009). Functional imaging of the human superior colliculus: an optimised approach. Neuroimage 47, 1620-1627.

Wurtz, R. H., and Albano, J. E. (1980). Visual-motor function of the primate superior colliculus. Annu. Rev. Neurosci. 3, 189-226.

Zambarbieri, D., Beltrami, G., and Versino, M. (1995). Saccade latency toward auditory targets depends on the relative position of the sound source with respect to the eyes. Vision Res. 35, 3305-3312.

Conflict of Interest Statement: The authors declare that the research was conducted in the absence of any commercial or financial relationships that could be construed as a potential conflict of interest.

Received: 13 June 2010; paper pending published: 17 June 2010; accepted: 24 September 2010; published online: 01 November 2010.

Citation: Krebs RM, SchoenfeldMA, Boehler CN, Song AW and WoldorffMG (2010) The saccadic re-centering bias is associated with activity changes in the human superior colliculus. Front. Hum. Neurosci. 4:193. doi: 10.3389/fnhum.2010.00193

Copyright (c) 2010 Krebs, Schoenfeld, Boehler, Song and Woldorff. This is an open-access article subject to an exclusive license agreement between the authors and the Frontiers Research Foundation, which permits unrestricted use, distribution, and reproduction in any medium, provided the original authors and source are credited. 